\title{
QCD NLO with Powheg matching and top threshold matching in WHIZARD
}

\author{
Jürgen Reuter* \\ DESY Theory Group, Notkestr. 85, 22607 Hamburg, Germany \\ E-mail: juergen.reuter@desy.de
}

Fabian Bach

European Commission, Eurostat, 2920 Luxembourg, Luxembourg

E-mail: fabian.bach@t-online.de

\section{Bijan Chokoufé Nejad}

DESY Theory Group, Notkestr. 85, 22607 Hamburg, Germany

E-mail: bijan.chokoufeddesy.de

\section{Wolfgang Kilian}

University of Siegen, Emmy-Noether-Campus, Walter-Flex-Str. 3, 57068 Siegen, Germany

E-mail: kilian@physik.uni-siegen.de

\section{Maximilian Stahlhofen}

PRISMA Cluster of Excellence, Institute of Physics, Johannes Gutenberg University, Staudingerweg 7, 55128 Mainz, Germany \& DESY Theory Group, Notkestr. 85, 22607

Hamburg, Germany

E-mail: mastahlheuni-mainz.de

\section{Christian Weiss}

DESY Theory Group, Notkestr. 85, 22607 Hamburg, Germany \& University of Siegen, Emmy-Noether-Campus, Walter-Flex-Str. 3, 57068 Siegen, Germany

E-mail: christian.weissedesy.de

We present the status of the automation of NLO processes within the event generator WHIZARD. The program provides an automated FKS subtraction and phase space integration over the FKS regions, while the (QCD) NLO matrix element is accessed via the Binoth Les Houches Interface from an externally linked one-loop program. Massless and massive test cases and validation are shown for several $e^{+} e^{-}$processes. Furthermore, we discuss work in progress and future plans. The second part covers the matching of the NRQCD prediction with NLL threshold resummation to the NLO continuum top pair production at lepton colliders. Both the S-wave and P-wave production of the top pair are taken into account in the resummation. The inclusion in WHIZARD allows to study more exclusive observables than just the total cross section and automatically accounts for important electroweak and relativistic corrections in the threshold region.

12th International Symposium on Radiative Corrections (Radcor 2015) and LoopFest XIV (Radiative Corrections for the LHC and Future Colliders)

15-19 June, 2015

UCLA Department of Physics \& Astronomy Los Angeles, USA 


\section{Introduction}

Monte Carlo event generators are indispensable tools for the comparison of data from high energy physics experiments with theory predictions. They should reflect the highest possible degree of precision from calculations in perturbative quantum field theory. In this proceedings article we describe several facets of the inclusion and automation of such higher-order calculations into the code WHIZARD. WHIZARD [1] is a multi-purpose event generator, that comes with its own powerful (tree-level) matrix element generator $\mathrm{O}^{\prime}$ Mega [2]. $\mathrm{O}^{\prime}$ Mega generates matrix elements for arbitrary processes either as Fortran 95/2003 code that is compiled or as byte code commands that are interpreted by a virtual machine (OVM) [3]. Integration is performed using a multi-channel adaptive Monte-Carlo integration using its subpackage VAMP [4]. As WHIZARD has been used for a long time as a major workhorse for lepton collider simulations, it contains the special tool CIRCE1/2 [5] that allows for the simulation of lepton collider beamstrahlung.

Especially at hadron colliders like the Large Hadron Collider (LHC), but also at high-energy lepton colliders, a precise treatment of QCD effects is indispensable. Color information is treated in WHIZARD using the color flow formalism [6]. WHIZARD comes with its own QCD parton showers, a $k_{T}$-ordered shower and an analytic parton shower [7]. WHIZARD ships with the final Fortran version of the Pythia 6 package [8] for hadronization, whose parton showers can be used alternatively to the internal ones. Direct interfaces to the standard event formats (like LHE, HepMC, LCIO, StdHEP) do exist, as well as interfaces for jet clustering and parton distribution functions. The most prominent PDFs are directly included without the need to take them from an external library. WHIZARD has a big tradition for studies of beyond the Standard Model physics (e.g. $[9,10,11,12,13,14,15,16])$, we will here concentrate on the (automated) inclusion of higher order (QCD) corrections to SM processes.

\section{Automation of QCD NLO corrections}

In order to be able to make reliable quantitative predictions for any kind of process, one has to include higher orders in the perturbative series. For decades, this has been (and still is) a tedious job, but in order to facilitate the procedure and to get to final results quicker, a high degree of standardization and even automation is highly desirable. In order to get a finite result that can be treated in a Monte Carlo integration, the soft and collinear (infrared) singularities as well as the ultraviolet divergences have to be treated before getting into phase space integration. The latter is done by means of renormalization in the virtual matrix elements. The former ones cancel in final states that are inclusive enough due to the KLN theorem [17, 18]. However, for this to happen, Born processes and virtual corrections have to be combined with real radiation corrections. In order to make the pieces that depend on final states of different multiplicites independently finite, so called subtraction formalisms have been invented. Here, the soft and collinear divergent regions are subtracted from the real emission diagrams to yield a finite result, while the same subtraction terms integrated analytically over the phase space of the emitted parton is added to the sum of Born and virtual contributions to make them finite as well. After this, all parts are finite and can be treated within the Monte Carlo integration.

\footnotetext{
* Speaker.
} 
The first attempt to include (virtual) next-to-leading order (NLO) corrections in WHIZARD was in the context of QED corrections to production of charginos at the ILC [19, 20] not using a subtraction formalism, but indeed a phase-space slicing plus photon resummation to obtain positive-weight NLO events. Virtual QCD corrections have for the first time been included in WHIZARD using Catani-Seymour subtraction [21] in [22, 23] for the process $p p \rightarrow b \bar{b} b \bar{b}+X$. This was using external virtual matrix elements from (the predecessors of) the Gosam package [24], and a hard-coded subtraction tailor-made for this specific process. Since version 2.2 of WHIZARD a preliminary version of the automated implementation of FKS matching $[25,26]$ has been published. According to the FKS prescription, for all possible singular regions, subtraction terms with
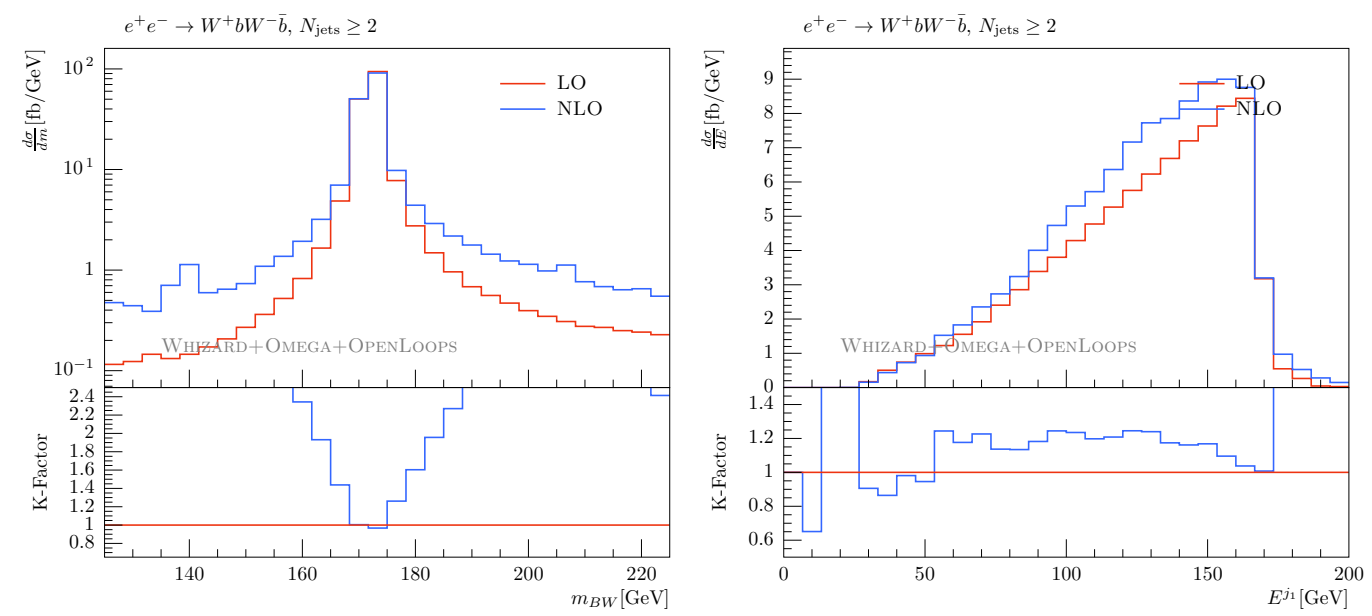

Figure 1: Resonant and non-resonant top pair production, $e^{+} e^{-} \rightarrow W^{+} b W^{-} \bar{b}$ at a future ILC at $\sqrt{s}=500$ $\mathrm{GeV}$. The left plot shows the $W b$ invariant mass distribution (where $b$ is the jet that contains the $b$ quark), while the plot on the right-hand side shows the energy of the hardest jet. Red lines are LO, blue ones NLO, respectively.

their corresponding phase space mappings are being generated. It automatically takes into account the color-correlated matrix elements for soft subtractions, and spin-correlated matrix elements for the collinear subtractions. External virtual matrix elements can be taken either from Gosam or OpenLoops [27] interfaced via the Binoth Les Houch Accord (BLHA) [28, 29]. The settings for NLO processes can be directly steered via commands within the SINDARIN command language of WHIZARD. A BLHA contract file to external one-loop providers is then automatically created by WHIZARD. Matrix elements from Gosam and OpenLoops have been checked against each other. Many different processes with uncolored initial states have been validated, like $e^{+} e^{-} \rightarrow q \bar{q}$, $e^{+} e^{-} \rightarrow q \bar{q} g, e^{+} e^{-} \rightarrow \ell^{+} \ell^{-} q \bar{q}, e^{+} e^{-} \rightarrow \ell^{+} v_{\ell} q \bar{q}, e^{+} e^{-} \rightarrow t \bar{t}, e^{+} e^{-} \rightarrow t W^{-} b, e^{+} e^{-} \rightarrow W^{+} b W^{-} \bar{b}$, $e^{+} e^{-} \rightarrow t \bar{t} H$, and $e^{+} e^{-} \rightarrow W^{+} b W^{-} \bar{b} H$. Cuts can be applied at the level of the clustered objects for the NLO processes. Fig. 1 shows as prime example differential distributions for the resonant and non-resonant top pair production for a $500 \mathrm{GeV}$ ILC. Virtual matrix elements have been taken from OpenLoops. The plot on the left-hand side shows the $W b$ invariant mass distribution which clearly shows the interference with the non-resonant background at NLO (this depends, of course, on the parameters of the jet clustering algorithm). The right hand side shows the energy distribution of the hardest jet in the final state. As the ILC will always run with beam polarization [30], WHIZARD can handle polarized cross sections at the LO, but also at the NLO level. Correspond- 
ing demands for polarized virtual matrix elements have to be stated in the BLHA contract file. This is beyond the standard BLHA convention and at the moment can be only processed with OpenLoops. It is also easily possible to integrate NLO processes that are not happening in the
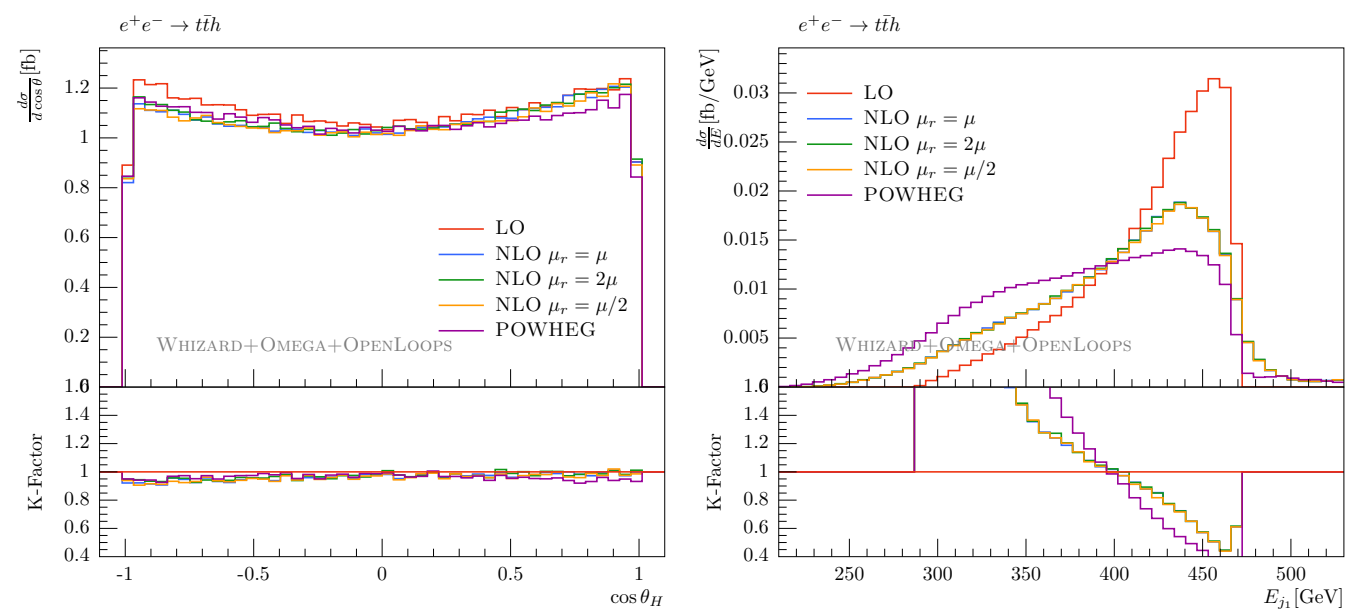

Figure 2: Angular distribution of the Higgs boson and energy distribution of the leading jet in the process $e^{+} e^{-} \rightarrow t \bar{t} H$ at $\sqrt{s}=1000 \mathrm{GeV}$. The dark magenta curve shows the POWHEG matching.

lab system due to initial state (photon) radiation or beamstrahlung. Also, decay processes can be integrated and simulated at the NLO-level, where the automated subtraction has to take care to generated subtraction terms and phase space in such a way as to maintain the mass of the decaying particle. This is important to have widths for resonant NLO processes in the same scheme/setup, as e.g. in the top threshold simulation in the next section. In the case that matrix elements contain narrow resonances decaying into colored objects, e.g. $Z \rightarrow q \bar{q}$ or $H \rightarrow b \bar{b}$, then soft and collinear radiation at NLO can drag final state particles either away from a resonance or push them towards a resonance. Both cases leads to a mismatch within the subtraction terms and a usually awful convergence of the Monte Carlo integration. Ref. [31] proposed a method to remedy this problem which has been applied to a specific process and by using a hard-coded resonance history in that reference. WHIZARD now has an automated implementation of this algorithm that generates the necessary resonance history. This will be included in the upcoming version WHIZARD 2.3. This formalism is particularly useful for the application of matrix element-parton shower matching methods at NLO, like e.g. POWHEG matching [32]. An automatic implementation of POWHEG matching is implemented in WHIZARD that combines (matches) the NLO fixed-order matrix element with the parton shower in such a way that the hardest emission is correctly described by the NLO matrix element. In Fig. 2 we show the process $e^{+} e^{-} \rightarrow t \bar{t} H$ for $\sqrt{s}=1000 \mathrm{GeV}$ at LO, at NLO using different scale choices and the POWHEG-matched result. As expected, the more inclusive angular distribution of the Higgs boson is rather insensitive to the QCD corrections, while the energy distribution of the leading jet depends strongly on it. Particularly, the Sudakov suppression at high energy values can be observed.

Before work in progress and future plans on the automation of fixed order NLO will be discussed in the summary, Sec. 4, we describe the implementation and matching of threshold resummation for the lepton collider top threshold and the continuum fixed-order NLO calculation. 


\section{Top Threshold Matching at Lepton Colliders}

High-energy lepton colliders that can be operated at or above the top threshold allow for the most precise method to measure the mass of the top quark known to date. In particular they allow for a direct extraction of the mass in a appropriate (short distance) scheme that is free of QCD renormalon uncertainties. For this, a threshold scan is performed as relatively inclusive measurement, and from the fit of the cross section line-shape to data the top mass can be extracted with an uncertainty well below $100 \mathrm{MeV}$. On the other hand, due to cuts and tagging in the final state, the measurement might not be as inclusive as anticipated, and differential quantities might allow for further improvements of the measurements at the top threshold. In particular this may be relevant for the precise measurement of other important (SM) parameters like the top width (i.e. $V_{t b}$ ), the strong coupling $\alpha_{s}$ or the top Yukawa coupling. Hence, it is desirable to have a Monte Carlo prediction for the threshold region. Furthermore, future lepton colliders might run at energies close to, but a bit off threshold (cf. e.g. the $380 \mathrm{GeV}$ staging from the CLIC study group). Therefore a smooth transition (matching) between the resummed threshold prediction and the fixed order prediction at large energies is required.

The top quark decays through the electroweak interaction before it hadronizes, but at the top threshold the attractive top quark potential leaves a remnant of a (1S) toponium bound state. The bound state effects are reflected by Coulomb singular terms $\propto\left(\alpha_{s} / v\right)^{n}$ in the perturbative expansion of the cross section, where $v \sim \alpha_{s} \sim 0.1$ is the relative velocity of the top quarks close to threshold. In addition to the Coulomb singularities, large logarithms $\propto \ln ^{n} v$ invalidate conventional fixed-order perturbation theory in the threshold region. These singular terms can be systematically resummed in vNRQCD [33], an effective field theory where modes at the hard scale, $m_{t}$, are integrated out. (Further scales in the theory are the soft scale, given by the the momentum, $m_{t} v$, and the ultrasoft scale, $m_{t} v^{2}$, given by the kinetic energies of the tops).

We implement the vNRQCD resummation in the Monte Carlo process by replacing the SM $t t \gamma, t t Z$ vertices by corresponding ( $\mathrm{S}$-wave and $\mathrm{P}$-wave) non-relativistic form factors at next-toleading logarithmic (NLL) order using the TOPPIK code [35]. At the same time we include the full set of relativistic QCD corrections at NLO, respectively. That is to say, we consistently add the terms beyond NLO from the NLL threshold resummation to the fixed order result. For technical details of the vNRQCD calculations, cf. [36, 37, 38, 39]. The matching between the threshold and the (relativistic) NLO prediction is performed by switching off the resummation above the threshold and expanding the resummed result. For onshell top quarks it is quite clear how to perform this as the complete amplitude can be simply multiplied with the form factor. For the nonresonant contributions in the process $e^{+} e^{-} \rightarrow W^{+} b W^{-} \bar{b}$ it is quite nontrivial due to the interference of threshold-enhanced terms with the non-resonant background. To maintain electroweak gauge invariance in the combination of the form factor and the QCD NLO corrections of the decay, we factorize production and decay in this contribution (similar to the pole approximation).

As consistency check we found agreement between the numerical WHIZARD implementation and the analytic non-relativistic vNRQCD result in the limit $\alpha_{s} \rightarrow 0$ for small $v$. Furthermore, in the threshold region, the Born prediction with an insertion of the form factor expanded to $\mathscr{O}\left(\alpha_{s}\right)$ should coincide with the fixed-order NLO cross section, because the vNRQCD form factor contains the dominant (non-vanishing) terms as $v \rightarrow 0$. This can be observed by the nice agreement of the 


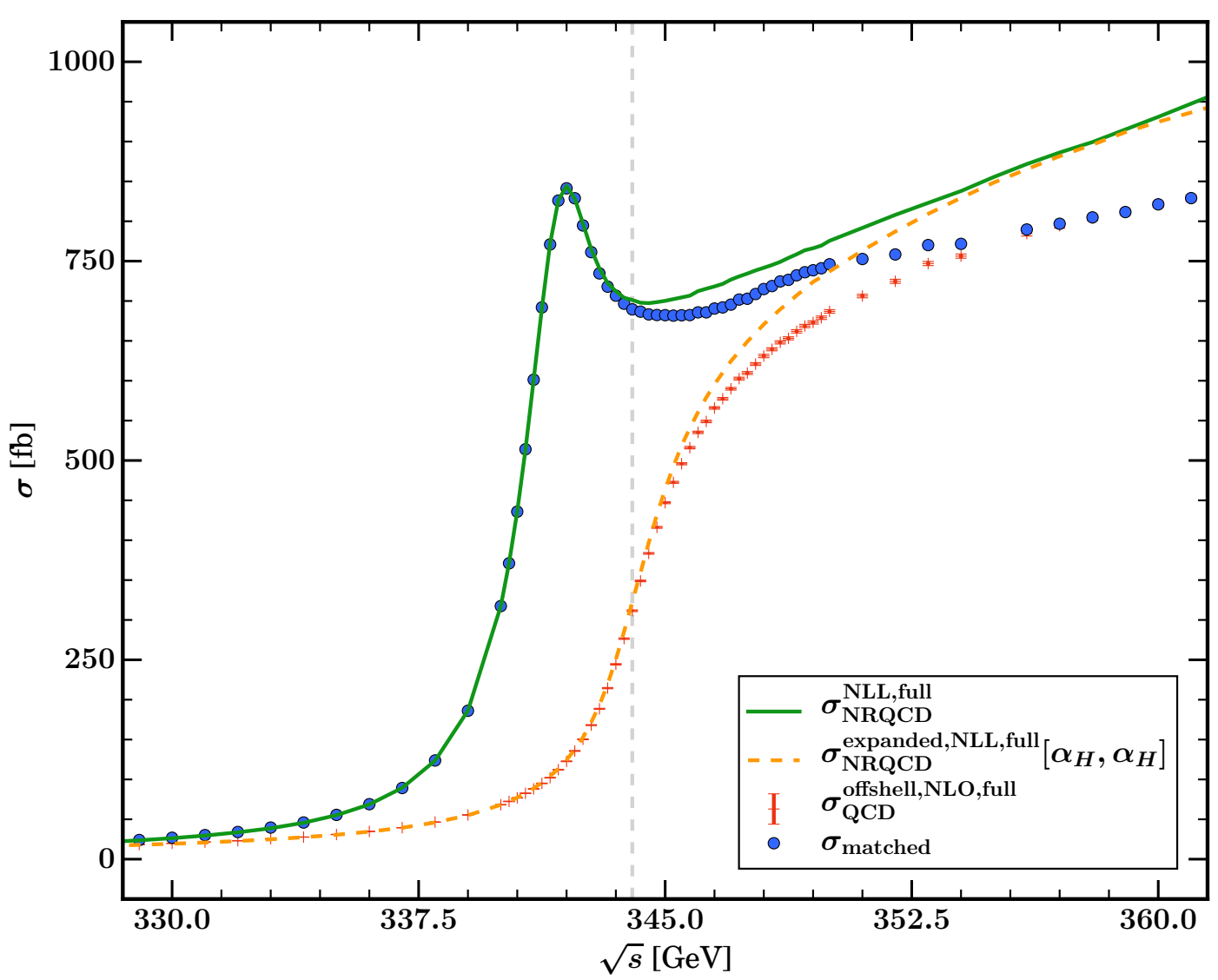

Figure 3: Matching of the NLL resummed threshold prediction to the fixed-order NLO QCD continuum for the total cross section of the process $e^{+} e^{-} \rightarrow W^{+} b W^{-} \bar{b}$. The red points are the NLO cross section values, the green curve corresponds to the inclusion of the NLL threshold resummed form factor into the Born process. The dashed orange curve corresponds to the latter result with the form factor expanded to first order in $\alpha_{s}$ and evaluated at the hard scale. (The two values of $\alpha_{s}$ are used in the hard matching coefficient and the Coulomb Green function of the form factor, respectively.) The blue dots show the result of our (preliminary) matching procedure. For this plot we have chosen $m_{t}=172 \mathrm{GeV}$ as well as the other parameters of Table 1 in [34].

orange curve and the red dots in Fig. 3 for $\sqrt{s}<345 \mathrm{GeV}$. The green curve shows the prediction corresponding to the insertion of the full NLL resummed form factor into the Born process. As the blue dots we show the final cross section with a matching prescription to interpolate between the non-relativistic threshold region and the continuum. The resummation in the form factor has to be switched off at large energies where the expansion in $v$ is no longer valid.

\section{Summary and Outlook}

This article gives a status report on the automation of NLO QCD corrections within the event generator WHIZARD. While virtual matrix elements are taken from external libraries or programs, subtraction terms are automatically generated for arbitrary SM processes according to the FKS 
algorithm, together with the corresponding partitioning of the phase space. Several cross checks, validations and examples have been given for uncolored initial states. The subtraction terms for initial-initial and initial-final singular regions are implemented and validated. Structure function reweighting for parton distribution functions in the initial state are up and working. Hence, all ingredients for automated NLO QCD processes for hadron colliders are present, but there have not been extensive enough checks and validations as of now.

Further steps in the more mid-term future will be the inclusion of QED and electroweak automated NLO corrections. Besides the POWHEG matching shown here, also merging schemes are being implemented in WHIZARD. The ultimate goal here is to have automated merging prescriptions for inclusive multi-jet samples.

A related, but conceptually different topic is the matching between the fixed-order QCD NLO matrix element for the continuum (off-shell) top quark pair production at lepton colliders to the NLL threshold production in VNRQCD. All technical and field-theoretical ingredients for this process are understood and being implemented. The implementation then allows for the simulation of more exclusive observables, and directly account for non-leading electroweak corrections within the implemented matrix elements. With our (preliminary) matching procedure the transition of the total cross section from the threshold to the continuum region looks satisfactory.

We plan to apply the same formalism for the $t t H$ production (off-shell) at the threshold, which this time is not driven by physical arguments but by the design energy of $500 \mathrm{GeV}$ of the International Linear Collider.

\section{Acknowledgments}

We would like to thank E. Bagnaschi, A. Denner, N. Greiner, A. Hoang, J. Lindert, and S. Pozzorini for their contribution to the projects summarized in this proceedings article, for providing and helping us with different codes like e.g. Gosam and Openloops, as well as for enlightening discussions on technical and physical details within perturbative quantum field theory. JRR wants to thank the organizers of Radcor/Loopfest 2015 for a great conference in the thrilling summer of Southern California.

\section{References}

[1] W. Kilian, T. Ohl and J. Reuter, Eur. Phys. J. C 71, 1742 (2011) [arXiv:0708.4233 [hep-ph]].

[2] M. Moretti, T. Ohl and J. Reuter, In *2nd ECFA/DESY Study 1998-2001* 1981-2009 [hep-ph/0102195].

[3] B. Chokoufe Nejad, T. Ohl and J. Reuter, Comput. Phys. Commun. 196, 58 (2015) doi:10.1016/j.cpc.2015.05.015 [arXiv:1411.3834 [physics.comp-ph]].

[4] T. Ohl, Comput. Phys. Commun. 120, 13 (1999) [hep-ph/9806432].

[5] T. Ohl, Comput. Phys. Commun. 101, 269 (1997) [hep-ph/9607454].

[6] W. Kilian, T. Ohl, J. Reuter and C. Speckner, JHEP 1210, 022 (2012) [arXiv:1206.3700 [hep-ph]].

[7] W. Kilian, J. Reuter, S. Schmidt and D. Wiesler, JHEP 1204, 013 (2012) [arXiv:1112.1039 [hep-ph]].

[8] T. Sjostrand, S. Mrenna and P. Z. Skands, JHEP 0605, 026 (2006) [hep-ph/0603175]. 
[9] W. Kilian, D. Rainwater and J. Reuter, Phys. Rev. D 71, 015008 (2005) [hep-ph/0411213].

[10] K. Hagiwara, W. Kilian, F. Krauss, T. Ohl, T. Plehn, D. Rainwater, J. Reuter and S. Schumann, Phys. Rev. D 73, 055005 (2006) [hep-ph/0512260].

[11] M. Beyer, W. Kilian, P. Krstonosic, K. Monig, J. Reuter, E. Schmidt and H. Schroder, Eur. Phys. J. C 48, 353 (2006) [hep-ph/0604048].

[12] A. Alboteanu, W. Kilian and J. Reuter, JHEP 0811, 010 (2008) [arXiv:0806.4145 [hep-ph]].

[13] J. Kalinowski, W. Kilian, J. Reuter, T. Robens and K. Rolbiecki, JHEP 0810, 090 (2008) [arXiv:0809.3997 [hep-ph]].

[14] W. Kilian, T. Ohl, J. Reuter and M. Sekulla, Phys. Rev. D 91, 096007 (2015) [arXiv:1408.6207 [hep-ph]].

[15] W. Kilian, T. Ohl, J. Reuter and M. Sekulla, arXiv:1511.00022 [hep-ph].

[16] J. S. Kim, J. Reuter, K. Rolbiecki and R. R. de Austri, arXiv:1512.06083 [hep-ph].

[17] T. Kinoshita, J. Math. Phys. 3, 650 (1962).

[18] T. D. Lee and M. Nauenberg, Phys. Rev. 133, B1549 (1964).

[19] W. Kilian, J. Reuter and T. Robens, Eur. Phys. J. C 48, 389 (2006) [hep-ph/0607127].

[20] T. Robens, J. Kalinowski, K. Rolbiecki, W. Kilian and J. Reuter, Acta Phys. Polon. B 39, 1705 (2008) [arXiv:0803.4161 [hep-ph]].

[21] S. Catani and M. H. Seymour, Nucl. Phys. B 485, 291 (1997) [Nucl. Phys. B 510, 503 (1998)] [hep-ph/9605323].

[22] T. Binoth, N. Greiner, A. Guffanti, J. Reuter, J.-P. Guillet and T. Reiter, Phys. Lett. B 685, 293 (2010) [arXiv:0910.4379 [hep-ph]].

[23] N. Greiner, A. Guffanti, T. Reiter and J. Reuter, Phys. Rev. Lett. 107, 102002 (2011) [arXiv:1105.3624 [hep-ph]].

[24] G. Cullen et al., Eur. Phys. J. C 74, no. 8, 3001 (2014) [arXiv:1404.7096 [hep-ph]].

[25] S. Frixione, Z. Kunszt and A. Signer, Nucl. Phys. B 467, 399 (1996) [hep-ph/9512328].

[26] R. Frederix, S. Frixione, F. Maltoni and T. Stelzer, JHEP 0910, 003 (2009) [arXiv:0908.4272 [hep-ph]].

[27] F. Cascioli, P. Maierhofer and S. Pozzorini, Phys. Rev. Lett. 108, 111601 (2012) [arXiv:1111.5206 [hep-ph]].

[28] T. Binoth et al., Comput. Phys. Commun. 181, 1612 (2010) [arXiv:1001.1307 [hep-ph]].

[29] S. Alioli et al., Comput. Phys. Commun. 185, 560 (2014) [arXiv:1308.3462 [hep-ph]].

[30] H. Baer et al., arXiv:1306.6352 [hep-ph].

[31] T. Ježo and P. Nason, JHEP 1512, 065 (2015) doi:10.1007/JHEP12(2015)065 [arXiv:1509.09071 [hep-ph]].

[32] P. Nason, JHEP 0411, 040 (2004) [hep-ph/0409146].

[33] M. E. Luke, A. V. Manohar and I. Z. Rothstein, Phys. Rev. D 61, 074025 (2000) doi:10.1103/PhysRevD.61.074025 [hep-ph/9910209]. 
[34] F. Bach and M. Stahlhofen, arXiv:1411.7318 [hep-ph].

[35] A. H. Hoang and T. Teubner, Phys. Rev. D 60, 114027 (1999) doi:10.1103/PhysRevD.60.114027 [hep-ph/9904468].

[36] A. H. Hoang and P. Ruiz-Femenia, Phys. Rev. D 74, 114016 (2006) doi:10.1103/PhysRevD.74.114016 [hep-ph/0609151].

[37] A. H. Hoang, C. J. Reisser and P. Ruiz-Femenia, Phys. Rev. D 82, 014005 (2010) doi:10.1103/PhysRevD.82.014005 [arXiv:1002.3223 [hep-ph]].

[38] A. H. Hoang and M. Stahlhofen, JHEP 1106, 088 (2011) doi:10.1007/JHEP06(2011)088 [arXiv:1102.0269 [hep-ph]].

[39] A. H. Hoang and M. Stahlhofen, JHEP 1405, 121 (2014) doi:10.1007/JHEP05(2014)121 [arXiv:1309.6323 [hep-ph]]. 